\title{
Accurate sequential detection of primary tumor and metastatic lymphatics using a temperature- induced phase transition nanoparticulate system
}

This article was published in the following Dove Press journal:

International Journal of Nanomedicine

18 June 2014

Number of times this article has been viewed

\author{
Keun Sang $\mathrm{Oh}^{\prime}$ \\ Ji Young Yhee ${ }^{2}$ \\ Dong-Eun Lee ${ }^{3}$ \\ Kwangmeyung $\mathrm{Kim}^{2}$ \\ Ick Chan Kwon ${ }^{2}$ \\ Jae Hong $\mathrm{Seo}^{4}$ \\ Sang Yoon $\mathrm{Kim}^{5}$ \\ Soon Hong Yuk ${ }^{1,4}$ \\ 'College of Pharmacy, Korea \\ University, Sejong, ${ }^{2}$ Biomedical \\ Research Center, Korea Institute \\ of Science and Technology, Seoul, \\ ${ }^{3}$ Advanced Radiation Technology \\ Institute, Korea Atomic Energy \\ Research Institute, Jeonbuk, \\ ${ }^{4}$ Biomedical Research Center, Korea \\ University Guro Hospital, Seoul, \\ ${ }^{5}$ Department of Otolaryngology, Asan \\ Medical Center, University of Ulsan, \\ College of Medicine, Seoul, Republic \\ of Korea
}

Correspondence: Soon Hong Yuk

College of Pharmacy, Korea University,

25 I I Sejongro, Sejong 339-700,

Republic of Korea

$\mathrm{Tel}+82448601612$

Fax +8244860 I606

Email shyuk@korea.ac.kr

\begin{abstract}
Primary tumor and tumor-associated metastatic lymphatics have emerged as new targets for anticancer therapy, given that these are difficult to treat using traditional chemotherapy. In this study, docetaxel-loaded Pluronic nanoparticles with Flamma ${ }^{\mathrm{TM}}$ (FPR-675, fluorescence molecular imaging dye; DTX/FPR-675 Pluronic NPs) were prepared using a temperature-induced phase transition for accurate detection of metastatic lymphatics. Significant accumulation was seen at the primary tumor and in metastatic lymph nodes within a short time. Particle size, maximum drug loading capacity, and drug encapsulation efficiency of the docetaxel-loaded Pluronic NPs were approximately $10.34 \pm 4.28 \mathrm{~nm}, 3.84 \mathrm{wt} \%$, and $94 \pm 2.67 \mathrm{wt} \%$, respectively. Lymphatic tracking after local and systemic delivery showed that DTX/FPR-675 Pluronic NPs were more potent in tumor-bearing mice than in normal mice, and excised mouse lymphatics showed stronger near-infrared fluorescence intensity on the tumor-bearing side than on the nontumor-bearing side at 60 minutes post-injection. In vivo cytotoxicity and efficacy data for the NPs demonstrated that the systemically administered NPs caused little tissue damage and had minimal side effects in terms of slow renal excretion and prolonged circulation in tumor-bearing mice, and rapid renal excretion in non-tumor-bearing mice using an in vivo real-time near-infrared fluorescence imaging system. These results clearly indicate that docetaxel-loaded Pluronic NPs could provide a strategy to achieve effective cancer therapy by simultaneous delivery to primary tumors, tumor lymphatics, and tumor-associated metastatic lymphatics.
\end{abstract}

Keywords: metastatic lymphatics, primary tumor targeting, lymphatic tracking, temperatureinduced phase transition, Pluronic nanoparticles

\section{Introduction}

Following on from the understanding that the first lymph nodes receive metastatic cancer cells via direct lymphatic drainage from the primary tumor, the sentinel lymph node (SLN) is recognized as a crucial element in cancer staging that limits the morbidity of treatment with excellent oncologic results. ${ }^{1-4}$ Moreover, as the clinical standard of care for cancer staging, tumor involvement of the SLN is identified using peritumoral injections of radiolabeled agents or imaging dyes (inorganic or organic), followed by SLN biopsy, which is an invasive surgical procedure. ${ }^{5-8}$ Therefore, there is a significant need to establish a noninvasive method for more accurate assessment of lymph node involvement with improved sensitivity and target specificity.

New macromolecular agents, including nanoparticles (NPs), have recently been used to image the lymphatics and SLN by magnetic resonance and optical techniques. ${ }^{9-11}$ Magnetic resonance imaging with ultrasmall superparamagnetic iron oxide NPs, which accumulate in the lymph nodes due to their prolonged circulation in the blood, has been used to reveal changes in the status of the SLN 
(ie, neoplasia). Gadolinium-labeled dendrimers with a coreshell structure have also been used successfully to visualize lymph node metastases. ${ }^{12-14}$ Further, indocyanine green, a near-infrared fluorescence (NIRF) probe approved by the US Food and Drug Administration, has exhibited potential in SLN mapping applications for breast cancer and gastric cancer. ${ }^{15-18}$ However, the very short half-life of indocyanine green in the blood (approximately 2-4 minutes) is its primary drawback as a molecular imaging probe. In order to improve these limitations, indocyanine green-incorporated polymer nanogels with a hydrodynamic diameter of approximately $30 \mathrm{~nm}$, which is optimal for uptake by lymph nodes, have been developed. ${ }^{19-22}$

In our previous reports, the formation of a nanocarrier with a core-shell structure was demonstrated through temperature-induced phase transitions in the melt state of a polyethylene glycol (PEG)/paclitaxel/Pluronic mixture. ${ }^{23}$ Liquid PEG (molecular weight 400) was used as a paclitaxel solubilizer, and the polymer that encapsulated the paclitaxel was composed of Pluronic F-68. For the preparation of docetaxel (DTX)-loaded Pluronic NPs, a soybean oil/Tween 80 mixture was used as the solubilizer instead of PEG in a temperatureinduced phase transition. ${ }^{24}$ The Pluronic NPs delivering paclitaxel or DTX showed improved antitumor efficacy compared with a Cremophor ${ }^{\circledR}$ EL-based paclitaxel formulation and a Tween 80/ethanol-based DTX formulation (Taxotere ${ }^{\circledR}$, Sanofi-aventis, Paris, France).$^{25}$ Interestingly, a significant decrease in diameter was observed in the Pluronic NPs formed with soybean oil/Tween 80 as the DTX solubilizer. The diameter of the optimized NPs changed as a function of the soybean oil/Tween 80 ratio, and their size distribution ranged from $10 \mathrm{~nm}$ to $20 \mathrm{~nm}$.

The lymphatic system is not easily accessible using conventional intravenous injections or infusions of chemotherapeutic agents, and this limits the amount of drug that can access the lymphatic tissues, including a metastatic lymph node. It has been reported that the primary controlling factor for the lymphatic uptake of injected NPs appears to be NP size, ie, NPs larger than $100 \mathrm{~nm}$ in diameter primarily remain at the injection site whereas those $10-80 \mathrm{~nm}$ in diameter are taken up well by the lymphatics. ${ }^{26-28}$

In this study, the suitability of DTX-loaded Pluronic nanoparticles with Flamma ${ }^{\mathrm{TM}}$ (FPR-675, a molecular imaging dye [DTX/FPR-675 Pluronic NPs]) as an efficient imaging modality and drug carrier for lymphatic cancer metastasis was confirmed because the particle size range was optimal for lymphatic drainage and retention of NPs in the lymph nodes. Based on cellular uptake behavior in various cell lines, an in vivo biodistribution study was conducted in order to confirm the increased tumor targetability and lymphatic tracking of the DTX/FPR-675 Pluronic NPs in tumor-bearing mice.

\section{Materials and methods Materials}

Pluronic F-68 [poly(ethylene oxide)-poly (propylene oxide)-poly (ethylene oxide)] triblock copolymer (molecular weight 8,350; $\left.(\mathrm{EO})_{79}(\mathrm{PO})_{28}(\mathrm{EO})_{79}\right)$ was obtained from BASF Company Ltd (Seoul, Republic of Korea). DTX (in anhydrous form) was purchased from Parling Pharma Tech Co Ltd (Shanghai, People's Republic of China). Polyoxyethylene sorbitan monooleate (Tween 80), soybean oil, and thiazolyl blue tetrazolium bromide (MTT) were purchased from Sigma (St Louis, MO, USA). FPR-675 dye was purchased from Bioacts (Incheon, Republic of Korea). Bovine aortic endothelial cells, murine macrophages (Raw264.7), and squamous cell carcinoma (SCC-7) cells were purchased from the American Type Culture Collection (Rockville, MD, USA). Roswell Park Memorial Institute 1640 medium, Dulbecco's Modified Eagle's Medium (low glucose), fetal bovine serum, and Dulbecco's phosphate-buffered saline were purchased from WelGENE Inc (Daegu, Republic of Korea). All experiments with live animals were performed in compliance with the relevant laws and institutional guidelines of the Korea Institute of Science and Technology, and institutional committees approved the experiments. BALB/c nude mice (aged 5 weeks, 20-25 g, male) and athymic nude mice (aged 5.5 weeks, 20-25 g, male) were purchased from Orient Bio Inc (Gyeonggi-do, Republic of Korea). All other chemicals were analytical grade and used without further purification.

\section{Methods}

\section{Preparation of the DTX/FPR-675 Pluronic NPs}

The DTX/FPR-675 Pluronic NPs were prepared by temperature-induced phase transition. In order to form a drugloaded phase at room temperature, $300 \mathrm{mg}$ of liquid soybean oil/Tween 80, $1 \mathrm{mg}$ of FPR-675 (loading amount $0.0033 \mathrm{wt} \%$ ), and $20 \mathrm{mg}$ of DTX (loading amount $6.25 \mathrm{wt} \%$ ) were mixed, and that mixture was subsequently mixed with $200 \mathrm{mg}$ of Pluronic F-68. As the temperature was increased to $60^{\circ} \mathrm{C}$, the mixture melted into a liquid phase. Equilibrium was maintained at $60^{\circ} \mathrm{C}$ for 10 minutes with stirring, and the liquid mixture was then sufficiently cooled to $0^{\circ} \mathrm{C}$ for 10 minutes to induce the phase transition. For in vitro and in vivo NIRF imaging, the FPR-675 dye was mixed with a soybean oil/Tween 80 / DTX mixture to observe lymphatic tracking in various organs 
and tumor distributions of the DTX/FPR-675 Pluronic NPs in SCC-7 tumor-bearing mice.

To remove the unloaded DTX, the NPs prepared by temperature-induced phase transition were mixed with distilled deionized water using a shaker under mild conditions at room temperature for 30 minutes. The dispersed NPs were then centrifuged at 3,000 rpm for 10 minutes followed by lyophilization or stored at $0^{\circ} \mathrm{C}$ for other experiments. To evaluate the physicochemical characteristics of the DTX/FPR-675 Pluronic NPs, a predetermined amount of distilled deionized water or phosphate-buffered saline ( $\mathrm{pH}$ 7.4) was added to the dried sample and the NPs were freely dispersed in mild mixing conditions in each solution without specific mechanical assistance such as sonication.

\section{Particle size and morphology of the DTX/FPR-675 Pluronic NPs}

The average diameter, size distribution, and zeta potential of the NPs $(1 \mathrm{mg} / \mathrm{mL}$ of NPs dispersed in phosphate-buffered saline) were measured by dynamic light scattering (Zetasizer Nano ZS90, Malvern Instruments Ltd, Malvern, UK) at $632.8 \mathrm{~nm}$ and $25^{\circ} \mathrm{C}$. Transmission electron micrographs were also taken to observe the morphology of the DTX/ FPR-675 Pluronic NPs. For the transmission electron microscopic measurements, the freeze-dried NPs were dispersed in distilled deionized water to obtain a solution of $0.1 \mathrm{wt} \%$. In order to prepare a sample, each solution was dropped on a 300 mesh copper grid coated with carbon and then dried at $25^{\circ} \mathrm{C}$ in a vacuum oven for 24 hours. Subsequently, the sample was negatively stained with $2 \mathrm{wt} \%$ uranyl acetate solution and dried. The samples were examined using an H-7600 microscope (Hitachi, Tokyo, Japan) operated at $100 \mathrm{kV}$.

\section{Release behavior of DTX from the DTX/FPR-675 Pluronic NPs}

In order to measure the release profile of DTX from the DTX/FPR-675 Pluronic NPs, 20 mg of NPs were dispersed in $3 \mathrm{~mL}$ of phosphate-buffered saline and placed in a dialysis bag (Spectrum ${ }^{\circledR}$, Rancho Dominquez, CA, USA; molecular weight cutoff 12,000-14,000), which was immersed in $15 \mathrm{~mL}$ of phosphate-buffered saline ( $\mathrm{pH} 7.4$ ) containing $0.1 \%$ (v/v) Tween 80 for sink conditions. The experimental setup was placed in a shaking water bath maintained at $37^{\circ} \mathrm{C}$ and was shaken horizontally at $150 \mathrm{rpm}$. At predetermined time intervals, $1.5 \mathrm{~mL}$ aliquots of the release medium (phosphatebuffered saline) were withdrawn and the total release medium was replaced with $15 \mathrm{~mL}$ of fresh phosphate-buffered saline to maintain sink conditions.
Quantification of the released DTX was determined using reverse-phase high performance liquid chromatography with a Capcell Pak-C18 column $(4.6 \times 250 \mathrm{~mm}, 5 \mu \mathrm{m})$ and an acetonitrile/water $(55 / 45, \mathrm{v} / \mathrm{v})$ mobile phase over 15 minutes at a flow rate of $1.0 \mathrm{~mL}$ per minute. The eluent was monitored by ultraviolet light absorption at $227 \mathrm{~nm}$. As a positive control, $10 \mathrm{mg}$ of DTX was dissolved in $0.27 \mathrm{~g}$ of Tween 80 (Sigma) and $0.2 \mathrm{~g}$ of ethanol (Tween 80/ethanolbased DTX formulation [Taxotere]) at room temperature. Prior to the release study, the free DTX stock solution was diluted with phosphate-buffered saline $(\mathrm{pH} 7.4)$ containing $0.1 \% \mathrm{v} / \mathrm{v}$ Tween 80 to a final concentration of about $250 \mu \mathrm{g}$ DTX per mL.

\section{In vitro cytotoxicity and cellular uptake of DTX/FPR-675 Pluronic NPs}

In order to evaluate the cytotoxicity and cellular behavior of the DTX/FPR-675 Pluronic NPs in various cell lines, bovine aortic endothelial cells (used as normal cells) were cultured in Dulbecco's Modified Eagle's Medium (low glucose; Gibco, Grand Island, NY, USA) containing 10\% (v/v) fetal bovine serum (Gibco) and 1\% (w/v) penicillin-streptomycin, and murine macrophages (Raw264.7) and squamous cell carcinoma (SCC-7) cells were cultured in Roswell Park Memorial Institute 1,640 medium containing 10\% (v/v) fetal bovine serum and $\%(\mathrm{w} / \mathrm{v})$ penicillin-streptomycin at $37^{\circ} \mathrm{C}$ in a humidified $5 \% \mathrm{CO}_{2}$ and $95 \%$ air atmosphere, respectively. The cells were seeded at a density of $5 \times 10^{3}$ cells per well in 96-well flat-bottomed plates and allowed to adhere overnight. The three types of cells were washed twice with fresh phosphate-buffered saline and incubated for 24 hours with various concentrations of the DTX/FPR-675 Pluronic NPs. The cytotoxicity of the DTX/FPR-675 Pluronic NPs was evaluated using a MTT-based assay. After 24 hours of incubation, the cell culture medium was eliminated. The cells were then washed twice with phosphate-buffered saline to eliminate the remaining drug-loaded Pluronic NPs. Next, $25 \mu \mathrm{L}$ of MTT solution ( $5 \mathrm{mg} / \mathrm{mL}$ in medium) was added to each well in the dark, and the cells were incubated for 2 hours at $37^{\circ} \mathrm{C}$. The precipitates (formazan crystals) were then added and dissolved by adding $200 \mu \mathrm{L}$ of dimethylsulfoxide. The absorbance was measured at $570 \mathrm{~nm}$ using a microplate reader (VersaMax ${ }^{\mathrm{TM}}$, Molecular Devices LLC, Sunnyvale, CA, USA). The percentages of viable cells were then determined by reduction of MTT relative to the negative control without the drug-loaded Pluronic NPs.

In order to compare the cellular uptake behavior of the DTX/FPR-675 Pluronic NPs, $1 \times 10^{4}$ cells were seeded on a 
dish with a cover slip and allowed to attach for 1 day. After cell attachment, the medium was replaced with $2 \mathrm{~mL}$ of serum with a culture medium containing the DTX/FPR-675 Pluronic NPs (5 mg of DTX) and then incubated for one hour. Next, the cells were washed twice with phosphate-buffered saline ( $\mathrm{pH} 7.4$ ) and fixed with a $4 \mathrm{v} / \mathrm{v} \%$ paraformaldehyde solution. For nuclear staining, the cells were incubated with $4^{\prime}, 6-$ diamidino-2-phenylindole ( $3 \mathrm{mmol}$ ) for 10 minutes at $25^{\circ} \mathrm{C}$, followed by several washes in phosphate-buffered saline. The intracellular localization of the DTX/FPR-675 Pluronic NPs was observed using an IX81-ZDC focus drift compensating microscope (Olympus, Tokyo, Japan), where the excitation and emission wavelengths were $673 \mathrm{~nm}$ and $692 \mathrm{~nm}$, respectively.

\section{Lymphatic tracking and tissue distribution of the DTX/FPR-675 Pluronic NPs}

The mice were anesthetized intraperitoneally using a solution containing $8 \mathrm{mg} / \mathrm{mL}$ ketamine (Ketalar ${ }^{\circledR}$, Panpharma, Fougères, France) and $0.8 \mathrm{mg} / \mathrm{mL}$ xylazine (Rompun ${ }^{\circledR}$, Bayer Pharma, Puteaux, France) at $0.015 \mathrm{~mL} / \mathrm{g}$ of body weight. The lymph node was examined after intradermal injection into the left forepaw pad with the DTX/FPR-675 Pluronic NPs in an aqueous dispersion ( $10 \mu \mathrm{L}, 1 \mathrm{mg} / \mathrm{mL}$ of DTX) using an NIRF image system. Images for lymphatic tracking were obtained at predetermined time points after NP injection using a $580 \mathrm{~nm}$ filtered charge coupled device camera under illumination at 460-490 nm, which was implemented in a high sensitivity imaging system (Olympus OV100) for data acquisition and analysis. In order to evaluate the distribution of NPs in lymphatic tissue, the lymph node (brachial) was obtained from normal mice and tumor-bearing mice and the obtained tissue was embedded in a Tissue Tek $^{\circledR}$ OCT compound (Sakura Finetek, Torrance, CA, USA) and cryosectioned at $8 \mu \mathrm{m}$ (CM1900 microtome, Leica). The fluorescent images were observed under a fluorescence microscope (Olympus BX51 and Zeiss Axioskop2 FS Plus).

\section{In vivo biodistribution, tumor targetability, and lymphatic tracking of the DTX/FPR-675 Pluronic NPs in tumor-bearing mice}

To observe the vivo biodistribution, SCC-7 tumor cells were induced in male nude mice (aged 5.5 weeks, Orient Bio Inc) by a subcutaneous injection of $1.0 \times 10^{6}$ cells suspended in cell culture (Roswell Park Memorial Institute 1,640 medium, 10\% fetal bovine serum, $1 \%$ antibiotic agent). When the tumor volume reached approximately $150-200 \mathrm{~mm}^{3}$, the mice received an intravenous injection of the DTX/FPR-675 Pluronic NPs with $10 \mathrm{mg}$ of DTX per $\mathrm{kg}$. In order to observe the tissue distribution, tumor targeting capability, and lymphatic tracking, each animal was positioned on an IVIS imaging system (Xenogen Corporation, Alameda, CA, USA) equipped with white-light excitation, charge coupled device camera-based fluorescence detection, and a filter set appropriate for nearinfrared-emitting FPR-675 dye molecules (emission detected at $700 \mathrm{~nm}$ ). The in vivo imaging system, consisting of a cooled charge coupled device camera mounted on a lighttight specimen chamber (dark box), a camera controller, a camera cooling system, and a Windows computer system, was used for data acquisition and analysis. The mice were placed in the specimen chamber mounted with the charge coupled device camera cooled to $-90^{\circ} \mathrm{C}$, with the field of view set at $25 \mathrm{~cm}$ above the sample shelf. The photon emission transmitted from the mice was measured. The gray scale photographic images and bioluminescence color images were superimposed using the Living Image ${ }^{\circledR}$ version 2.11 software overlay (Xenogen) and $\mathrm{IGOR}^{\circledR}$ image analysis software (version 4.02A, WaveMetrics, Lake Oswego, OR, USA). A region of interest was manually selected over the signal intensity. The area of the region of interest was kept constant and the intensity was recorded as maximum (photons $\cdot \mathrm{s}^{-1} \cdot \mathrm{cm}^{-2} \cdot \mathrm{sr}^{-1}$ [steradian]) within a region of interest. The mice, kept in an anesthetic state by inhalation of Gerolan ${ }^{\circledR}$ solution (enflurane as the active agent, Choong Wae Pharmaceuticals, Seoul, Republic of Korea), were automatically moved into the imaging chamber for scanning. The in vivo characteristics of the DTX/FPR-675 Pluronic NPs were confirmed by measuring the NIRF intensity in the SCC-7 tumor-bearing mice $(n=3)$. To compare the distribution in various lymphatic tissues of each sample, the mice were euthanized at 60 minutes postinjection. The various lymph nodes (superficial cervical, brachial, and inguinal) were dissected from the mice, and their fluorescence intensities were determined using a 12-bit charge coupled device camera (Image Station 4000 MM; Kodak, New Haven, CT, USA) equipped with a special C-mount lens and a FPR-675 bandpass emission filter (680-720 nm; Omega Optical, Brattleboro, VT, USA). Identical illumination settings (eg, lamp voltage, filter, exposure time) were used in all animal imaging experiments.

\section{Statistical analysis}

The data are expressed as the mean \pm standard deviation of at least three experiments. Data for the in vivo characteristics using an NIRF system were calculated using the region of interest function of the Analysis Workstation software package (ART Advanced Research Technologies Inc., Montreal, 
QC, Canada). All data processing was performed using the ORIGIN $^{\circledR} 8.0$ statistical software program (OriginLab Corporation, Northampton, MA, USA).

\section{Results}

Preparation and characterization of DTX/FPR-675 Pluronic NPs

To demonstrate the targeting and tracking of the DTX/ FPR-675 Pluronic NPs in the primary tumor and metastatic lymphatics, as described in Figure 1, various DTX/ FPR-675 Pluronic NPs were prepared with different soybean oil/Tween 80 ratios as a solubilizer for DTX and FPR-675. To optimize the composition of the DTX/FPR-675 Pluronic NPs, the soybean oil and Tween 80 mixtures were prepared with different weight ratios and characterized as shown in Figures 2A and 2B. Assessment confirmed stable dispersion of the DTX/FPR-675 Pluronic NPs with a soybean oil/Tween 80 ratio of 0.2 in the aqueous

A FPR-675
(NIR fluorescence dye)
(hydrophobic anti-cancer drug)

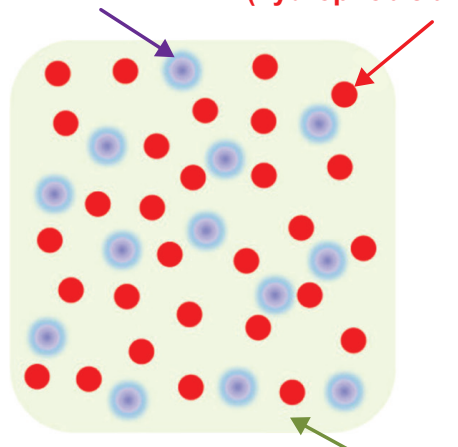

Soybean oil/Tween 80 mixtures

Docetaxel and FPR-675 dissolved in soybean oil/Tween 80 mixtures

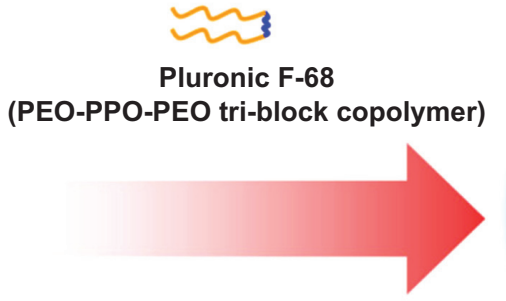

Heating and cooling under mild stirring

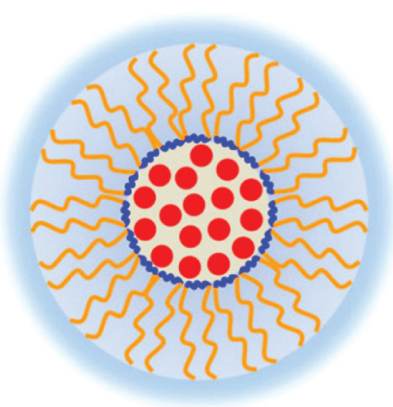

DTX/FPR-675 Pluronic NPs by Temperature-induced phase transition

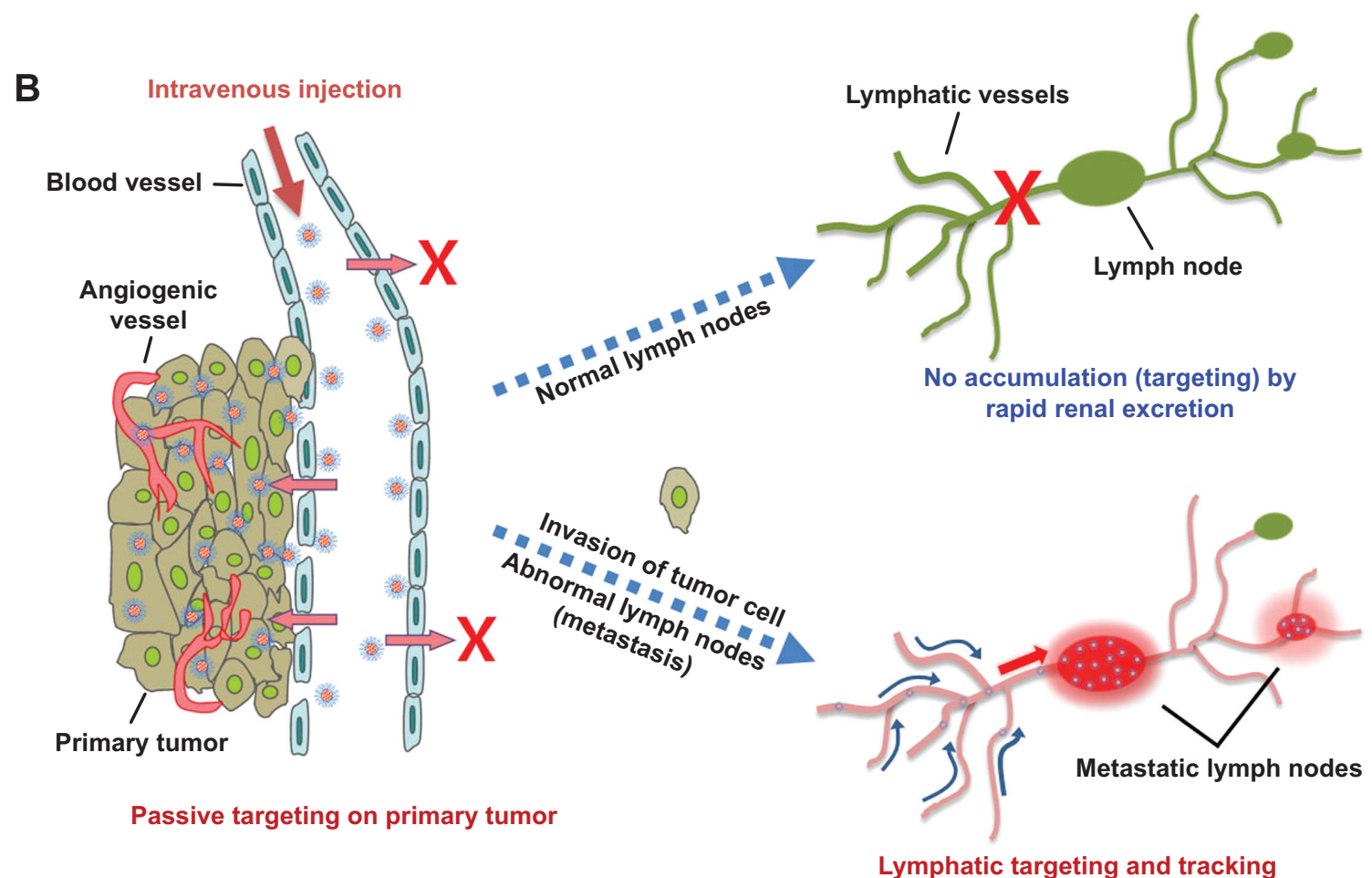

Figure I Schematic and expected behavior of DTX/FPR-675 Pluronic NPs.

Notes: (A) Schematic of DTX/FPR-675 Pluronic NPs according to temperature-induced phase transition and (B) expected behavior of NPs in blood vessels and lymphatic vessels. Abbreviations: DTX, docetaxel; NPs, nanoparticles; FPR-675, Flamma ${ }^{\text {TM }}$ fluorescence molecular imaging dye; NIR, near-infrared; PEO-PPO-PEO, poly(ethylene oxide)-poly (propylene oxide)-poly(ethylene oxide). 

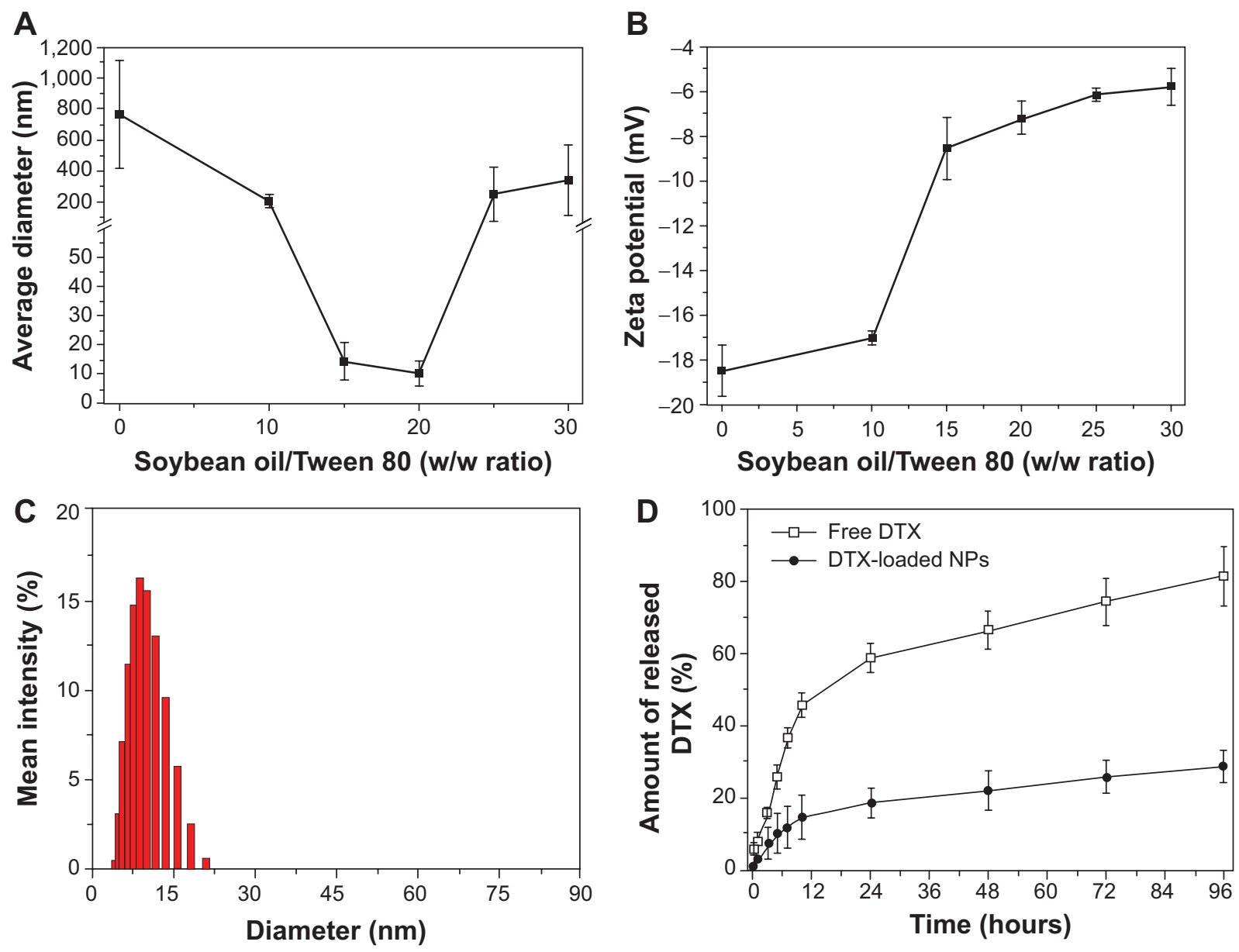

Figure 2 Characteristics of DTX/FPR-675 Pluronic NPs.

Notes: (A) Size changes and (B) zeta potential of DTX/FPR-675 Pluronic NPs prepared with different soybean oil/Tween 80 weight ratios. (C) Size distribution and morphology and (D) in vitro release behavior of DTX/FPR-675 Pluronic NPs. Data are presented as the mean $\pm s t a n d a r d ~ d e v i a t i o n ~(n=4)$.

Abbreviations: DTX, docetaxel; NPs, nanoparticles; FPR-675, Flamma ${ }^{\text {TM }}$ fluorescence molecular imaging dye; w, weight.

medium with a single distribution of $10.34 \pm 4.28 \mathrm{~nm}$ (Figure 2C). The maximum loading capacity and encapsulation efficiency of the NPs were $3.84 \pm 0.65 \mathrm{wt} \%$ and $94 \pm 2.67 \mathrm{wt} \%$, respectively.

The in vitro release profiles for the DTX/FPR-675 Pluronic NPs were measured at $\mathrm{pH} 7.4$ as shown in Figure 2D. The DTX/FPR-675 Pluronic NPs showed a sustained release pattern typical of that observed for drug release from a homogeneous polymer matrix, and differed from that found with free DTX. Therefore, DTX/FPR-675 Pluronic NPs of $10.34 \pm 4.28 \mathrm{~nm}$ were used throughout the experiments.

\section{In vitro cytotoxicity and cellular uptake} of DTX/FPR-675 Pluronic NPs

To compare the cytotoxicity and cellular uptake behavior of the DTX/FPR-675 Pluronic NPs, three cell types (normal [bovine aortic endothelial] cells, macrophage [Raw264.7] cells, and tumor [SCC-7] cells) were evaluated by MTT assay and fluorescent images (Figure 3).
Growth inhibition was found in all cells and the DTX/FPR-675 Pluronic NPs showed drug concentrationdependent cytotoxicity in the cell culture system. The DTX/FPR-675 Pluronic NPs had a more efficient cytotoxic effect on the SCC-7 tumor cells than on the bovine aortic endothelial cells and Raw264.7 cells.

To observe the cellular uptake behavior, the DTX/FPR675 Pluronic NPs were incubated for 60 minutes and compared by the fluorescence intensity of each cell (Figure 3B). When their subcellular localization was observed under a fluorescent microscope, the fluorescence from the DTX/FPR-675 Pluronic NPs was not in the nuclear compartment of the SCC-7 tumor cells. All cells treated with the DTX/FPR-675 Pluronic NPs exhibited a red fluorescence signal in the cytoplasm, and the intensity was particularly high in the SCC-7 tumor cells. In contrast, the bovine aortic endothelial cells and Raw264.7 cells showed very low fluorescence intensity, indicating the possibility of low unintended uptake of 

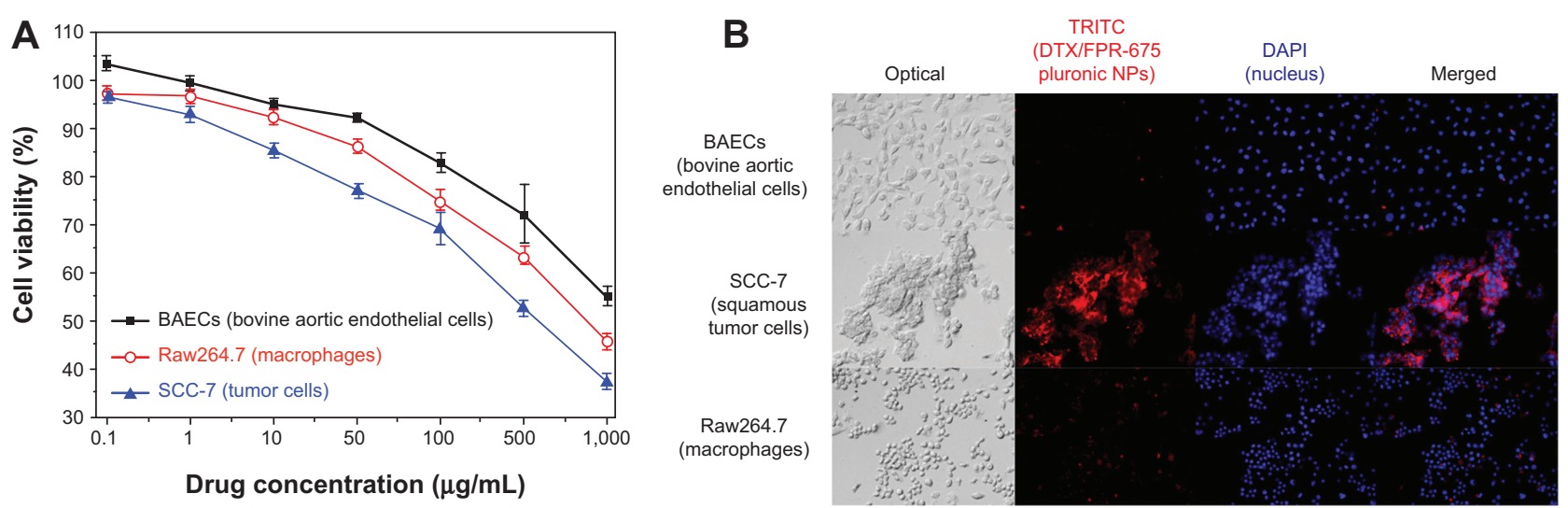

Figure 3 Cytotoxicity and in vitro cellular uptake of the NPs in various cells.

Notes: (A) In vitro cytotoxicity at various concentrations of the DTX/FPR-675 Pluronic NPs from $0.1 \mathrm{~g} / \mathrm{mL}$ to I,000 g/mL and (B) cellular behavior of the DTX/FPR-675 Pluronic NPs using normal cells (BAECs), macrophages (Raw264.7), and tumor (SCC-7) cells. Data are presented as the mean \pm standard deviation ( $\mathrm{n}=6$ ).

Abbreviations: BAECs, bovine aortic endothelial cells; DTX, docetaxel; NPs, nanoparticles; FPR-675, Flamma ${ }^{\text {TM }}$ fluorescence molecular imaging dye; TRITC, tetramethylrhodamine; DAPI, 4',6-diamidino-2-phenylindole.

the DTX/FPR-675 Pluronic NPs into normal cells and macrophages.

\section{Lymphatic tracking and tissue distribution of DTX/FPR-675 Pluronic NPs in normal}

\section{and tumor-bearing mice}

To compare the lymphatic accumulation and tissue distribution in two types of mice (non-tumor-bearing and tumorbearing), the lymph nodes were identified immediately after intradermal injection of DTX/FPR-675 Pluronic NPs into the forepaw pad of mice using a real-time NIRF imaging system. After injection, the DTX/FPR-675 Pluronic NPs in the tumorbearing mice were rapidly transported from the injection site to the lymphatics. The lymph nodes and lymphatic vessels were visualized within one minute and then were continually visualized for a minimum of 60 minutes (early diagnosis time), and then we gradually decreased the fluorescence intensity at the lymph node. However, in the normal mice, there was no transport of the DTX/FPR-675 Pluronic NPs to the lymphatics and the flow status was observed at the initial time due to injected pressure on the mouse forepaw pad (Figure 4A). Therefore, accurate sites for the lymphatics and lymphatic vessels were more intensely recognized in tumor-bearing mice. Likewise, quantitative analyses of the fluorescence intensity at the brachial node were performed for an extended time period, and higher fluorescence intensities were observed in tumor-bearing mice than in normal nontumor-bearing mice (Figure 4B). Further, fluorescence images from a frozen section of lymphatic tissue were observed to verify enhanced accumulation of DTX/FPR-675 Pluronic NPs in a tumor-bearing mouse. The DTX/FPR-675 Pluronic NPs accumulated in both the external and internal regions of the lymph node (Figure 4C).

\section{Biodistribution, tumor targetability, and lymphatic} tracking of DTX/FPR-675 Pluronic NPs

in tumor-bearing mice

Because the FPR-675 was physically entrapped in the NPs, the difference in biodistribution was observed between the DTX/FPR-675 Pluronic NPs and the freeFPR-675. ${ }^{29,30}$ Figure 5A demonstrates that the DTX/FPR-675 Pluronic NPs have stronger fluorescence intensity of a yellow and red color than the free FPR-675 in the superficial cervical node and brachial node until 60 minutes post-injection. In addition, the quantification data for each lymph node showed a significant difference and an approximately four-fold higher intensity than the free FPR-675, as shown in Figure 5B and 5C. Similar results were observed with the excised lymphatic tissues as presented in Figure 5D. Interestingly, the fluorescence intensity from the tumor-bearing side was more significantly different than that from the non-tumor-bearing side.

The whole body distribution behavior of the DTX/FPR675 Pluronic NPs in vivo was monitored using real-time NIRF imaging for up to 9 hours post-injection (Figure 6A). After intravenous injection, the DTX/FPR-675 Pluronic NPs showed strong fluorescence signals at the lymphatics (superficial cervical node and brachial node) from 3 hours post-injection. The fluorescence at the tumor site gradually increased after one hour post-injection, and the highest NIRF intensity in the tumor was observed at 7 hours post-injection. Figure 6A confirms that the experimental results presented in Figure 5 were maintained for extended periods of time.

The renal clearance of DTX/FPR-675 Pluronic NPs is presented in Figure 6B. ${ }^{31}$ Strong fluorescence intensity was observed in the bladder of the normal mouse at one hour postinjection and this indicates that the DTX/FPR-675 Pluronic 

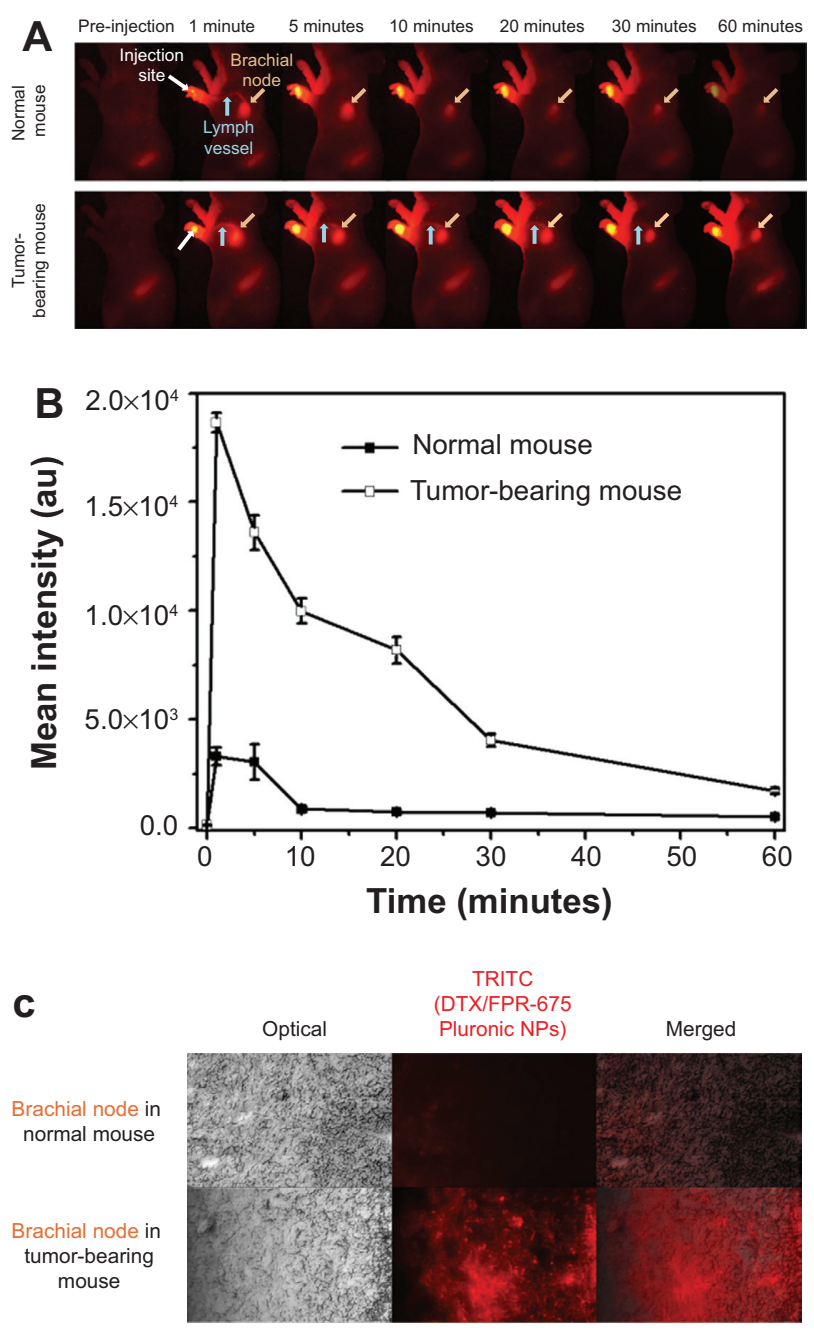

Figure 4 In vivo and ex vivo near-infrared fluorescence images of a lymph node with DTX/FPR-675 Pluronic NPs.

Notes: (A) In vivo real-time near-infrared fluorescence images and (B) fluorescence quantification data for a localized lymph node in a mouse $(n=4)$ within 60 minutes of being intradermally injected with DTX/FPR-675 Pluronic NPs (10 $\mu \mathrm{L}, 5 \mathrm{mg} / \mathrm{mL}$ DTX) in the right forepaw pad. The filter set has Cy5.5 emission $(700 \mathrm{~nm})$. (C) Fluorescence microscopic images of a dissected brachial lymph node from a non-tumor-bearing mouse (upper) and a tumor-bearing mouse (lower) with DTX/FPR-675 Pluronic NPs 60 minutes post-injection.

Abbreviations: DTX, docetaxel; NPs, nanoparticles; FPR-675, Flamma ${ }^{\mathrm{TM}}$ fluorescence molecular imaging dye; TRITC, tetramethylrhodamine.

NPs in normal mice were rapidly excreted from the body through the kidneys and bladder. However, minimal clearance of the DTX/FPR-675 Pluronic NPs was observed in the bladders of tumor-bearing mice.

\section{Discussion}

It is generally accepted that nanoparticulate systems with diameters of less than $200 \mathrm{~nm}$ can avoid uptake by the reticuloendothelial system, circulate in the bloodstream for a prolonged period, and consequently accumulate in the tumor region. Furthermore, if their size is less than $20-30 \mathrm{~nm}$, they can be utilized for lymphatic delivery. ${ }^{26-28}$
Based on previous studies, ${ }^{23,24}$ DTX/FPR-675 Pluronic NPs with a diameter of approximately $10.34 \pm 4.28 \mathrm{~nm}$ were prepared by temperature-induced phase transitions. Given their size, DTX/FPR-675 Pluronic NPs can be utilized as vehicles for lymphatic delivery of molecular imaging probes and chemotherapeutic agents. As shown in Figure 3, the DTX/FPR-675 Pluronic NPs were successfully delivered to the cytoplasm of SCC-7 tumor cells, which is the target site of the drug; however, minimal uptake was observed in other cells (normal [bovine aortic endothelial] cells and macrophages [Raw264.7]). It has been reported that the major factors affecting efficient tumor targeting of NPs have a close relationship with greater uptake of NPs into tumor cells than other cell types. ${ }^{32}$ Based on this previous report, the minimal cellular uptake of the NPs in normal cells can be explained. If the DTX/FPR-675 Pluronic NPs are delivered to the tumor site, they can be taken up by cancer cells efficiently and lymphatic delivery can be expected, as described in Figure 1. As presented in Figure 4, conclusive evidence for delivery of DTX/FPR-675 Pluronic NPs to the lymph nodes in tumor-bearing mice strongly supports the potential application of DTX/FPR-675 Pluronic NPs as a multifunctional nanocarrier for molecular imaging probes and chemotherapeutic drugs.

Because FPR-675 is physically entrapped in the NPs, release of FPR-675 is expected during systemic circulation of the NPs. The whole body distribution of the DTX/FPR-675 Pluronic NPs showed more enhanced accumulation at the lymph nodes (superficial cervical and brachial) than that of free FPR-675 (Figure 5A-C). This significant difference in biodistribution behavior between DTX/FPR-675 Pluronic NPs and free FPR-675 confirmed that the fluorescence from DTX/FPR-675 Pluronic NPs represented the tracking of NPs. Figure 5D indicates similar behavior of DTX/FPR-675 Pluronic NPs in the excised lymphatic tissue. In addition, a clear difference in the fluorescence intensity was observed between the tumor-bearing side and non-tumorbearing side in the tumor-bearing mice, indicating accurate tracking of metastatic lymphatics using DTX/FPR-675 Pluronic NPs. These results suggest that a biodistribution study using DTX/FPR-675 Pluronic NPs could provide information on the lymphatic tracking of NPs in a tumor environment.

The whole body distribution and renal clearance behavior of the DTX/FPR-675 Pluronic NPs were examined for extended periods, as shown in Figure 6. Figure 6A confirms that the experimental results presented in Figure 5 were maintained for extended periods of time. In the absence of abnormal (ie, tumor) tissue for enhanced permeation and retention, rapid renal clearance was confirmed in the normal 

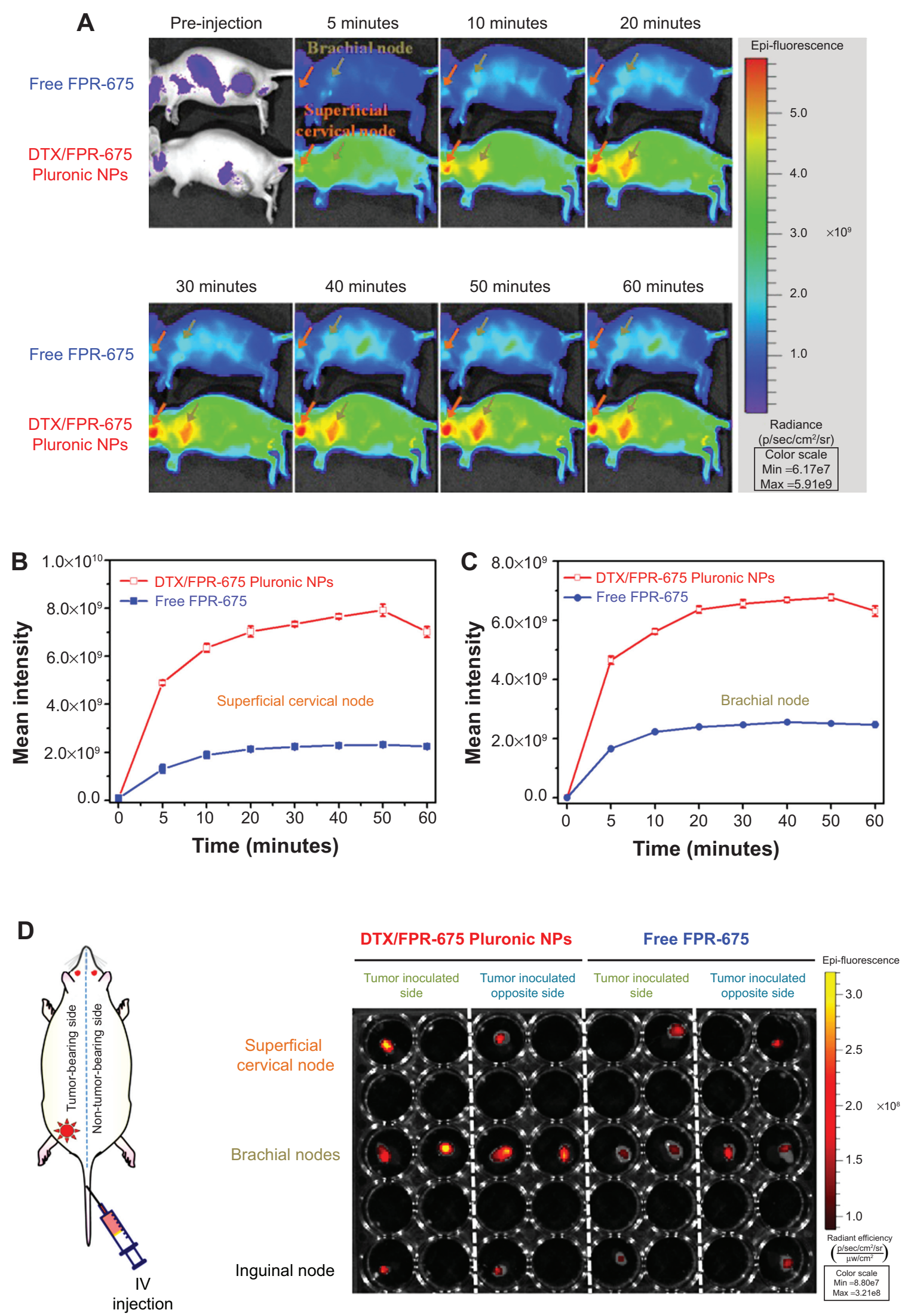

Figure 5 In vivo real-time and excised lymph node near-infrared fluorescence imaging of tumor-bearing mice injected with DTX/FPR-675 Pluronic NPs for lymph node imaging. Notes: In vivo whole body near-infrared fluorescence images of mice ( $n=4) 60$ minutes post-injection of (A) free FPR-675 (upper) and DTX/FPR-675 Pluronic NPs (lower). Comparison of near-infrared fluorescence intensity in (B) an excised superficial cervical (right) and a brachial (left) node and (C) near-infrared fluorescence images using DTX/FPR-675 Pluronic NPs. (D) Representative ex vivo fluorescence images of dissected lymph nodes (superficial cervical, brachial, and inguinal). Abbreviations: DTX, docetaxel; NPs, nanoparticles; FPR-675, Flamma ${ }^{\mathrm{TM}}$ fluorescence molecular imaging dye; IV, intravenous. 


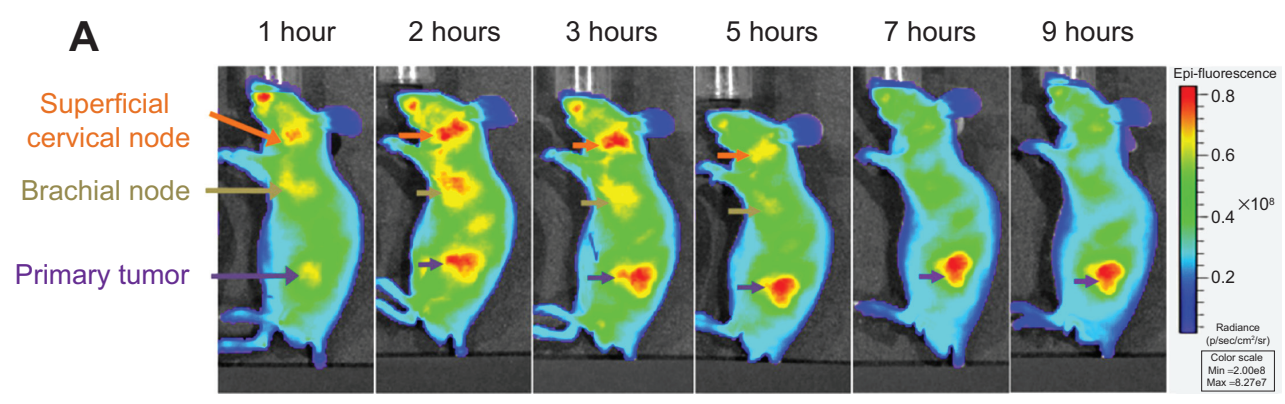

B

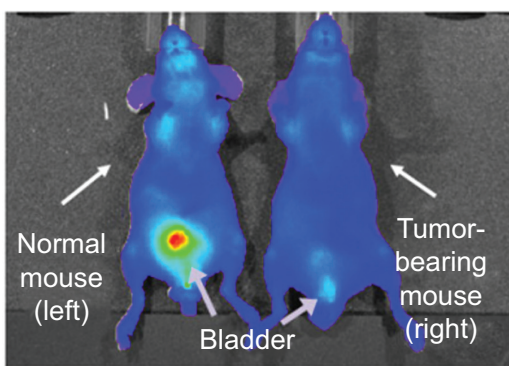

5 hours

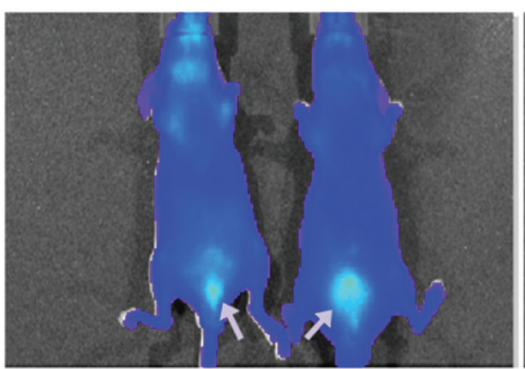

3 hours

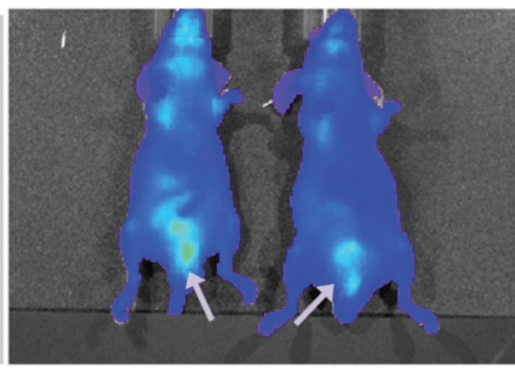

7 hours

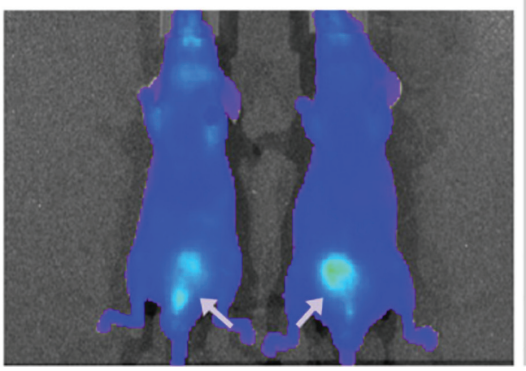

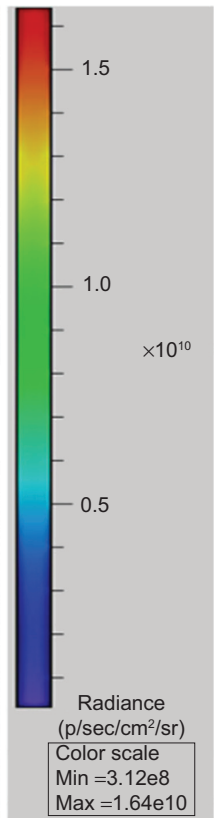

Figure 6 In vivo real-time whole body near-infrared fluorescence images and clearance of nanoparticles at different time points.

Notes: (A) In vivo real-time whole body near-infrared fluorescence images for extended periods. (B) Images of clearance via renal excretion.

mice through observation of strong fluorescence intensity in the bladder one hour post-injection (Figure 6B). These results also indicate prolonged circulation of DTX/FPR-675 Pluronic NPs in the blood, leading to enhanced tumor accumulation at the primary tumor and metastatic lymphatics. ${ }^{33-35}$

\section{Conclusion}

DTX/FPR-675 Pluronic NPs were prepared using selfassembled Pluronic NPs with soybean oil/Tween 80 mixtures, and their use for accurate detection of primary tumors and metastatic lymphatics was demonstrated. At the optimum composition of soybean oil/Tween 80 mixtures with DTX and FPR-675, the DTX/FPR-675 Pluronic NPs were prepared with a single distribution of diameter (approximately $10 \mathrm{~nm}$ ) and a clear difference in lymphatic accumulation was seen between normal and tumor-bearing mice. The preliminary results of this study demonstrated that DTX/FPR-675 Pluronic NPs may provide a strategy for improving cancer therapy through simultaneous delivery of molecular imaging probes and chemotherapeutic agents to the primary tumor, tumor lymphatics, and tumor-associated metastatic lymphatics.

\section{Acknowledgment}

This work was supported by National Research Foundation of Korea grants (20110027932, 2012028831, and 2013063969) funded by the Korean government.

\section{Disclosure}

The authors report no conflicts of interest in this work.

\section{References}

1. Lyman GH, Giuliano AE, Somerfield MR, et al. American Society of Clinical Oncology guideline recommendations for sentinel lymph node biopsy in early-stage breast cancer. J Clin Oncol. 2005;23(30):7703-7720.

2. Amersi F, Hansen NM. The benefits and limitations of sentinel lymph node biopsy. Curr Treat Options Oncol. 2006;7(2):141-151.

3. Schofield JB, Mounter NA, Mallett R, Haboubi NY. The importance of accurate pathological assessment of lymph node involvement in colorectal cancer. Colorectal Dis. 2006;8(6):460-470. 
4. Schulze T, Mucke J, Markwardt J, Schlag PM, Bembenek A. Long-term morbidity of patients with early breast cancer after sentinel lymph node biopsy compared to axillary lymph node dissection. J Surg Oncol. 2006;93(2):109-119.

5. Beri A, Janetschek G. Technology insight: radioguided sentinel lymph node dissection in the staging of prostate cancer. Nat Clin Pract Urol. 2006;3(11):602-610

6. Ting R, Aguilera TA, Crisp JL, et al. Fast 18F labeling of a near-infrared fluorophore enables positron emission tomography and optical imaging of sentinel lymph nodes. Bioconjug Chem. 2010;21(10):1811-1819.

7. Yokoyama J, Fujimaki M, Ohba S, et al. A feasibility study of NIR fluorescent image-guided surgery in head and neck cancer based on the assessment of optimum surgical time as revealed through dynamic imaging. Onco Targets Ther. 2013;6:325-330.

8. Kelderhouse LE, Chelvam V, Wayua C, et al. Development of tumortargeted near infrared probes for fluorescence guided surgery. Bioconjug Chem. 2013;24(6):1075-1080.

9. Saokar A, Braschi M, Harisinghani M. Lymphotrophic nanoparticle enhanced MR imaging (LNMRI) for lymph node imaging. Abdom Imaging. 2006;31(6):660-667.

10. Niu C, Wang Z, Lu G, et al. Doxorubicin loaded superparamagnetic PLGA-iron oxide multifunctional microbubbles for dual-mode US MR imaging and therapy of metastasis in lymph nodes. Biomaterials. 2013;34(9):2307-2317.

11. Wu Q, Chu M. Self-illuminating quantum dots for highly sensitive in vivo real-time luminescent mapping of sentinel lymph nodes. Int J Nanomedicine. 2012;7:3433-3443.

12. Kobayashi H, Kawamoto S, Bernardo M, Brechbiel MW, Knopp MV, Choyke PL. Delivery of gadolinium-labeled nanoparticles to the sentinel lymph node: comparison of the sentinel node visualization and estimations of intra-nodal gadolinium concentration by the magnetic resonance imaging. J Control Release. 2006;111(3):343-351.

13. Talanov VS, Regino CA, Kobayashi H, Bernardo M, Choyke PL, Brechbiel MW. Dendrimer-based nanoprobe for dual modality magnetic resonance and fluorescence imaging. Nano Lett. 2006;6(7): $1459-1463$.

14. Kobayashi H, Kawamoto S, Choyke PL, et al. Comparison of dendrimerbased macromolecular contrast agents for dynamic micro-magnetic resonance lymphangiography. Magn Reson Med. 2003;50(40):758-766.

15. Nimura H, Narimiya N, Mitsumori N, Yamazaki Y, Yanaga K, Urashima M Infrared ray electronic endoscopy combined with indocyanine green injection for detection of sentinel nodes of patients with gastric cancer. Br J Surg. 2004;91(5):575-579.

16. Kitai $\mathrm{T}$, Inomoto $\mathrm{T}$, Miwa $\mathrm{M}$, Shikayama $\mathrm{T}$. Fluorescence navigation with indocyanine green for detecting sentinel lymph nodes in breast cancer. Breast Cancer. 2005;12(3):211-215.

17. Akers WJ, Kim C, Berezin M, et al. Noninvasive photoacoustic and fluorescence sentinel lymph node identification using dye-loaded perfluorocarbon nanoparticles. ACS Nano. 2011;5(1):173-182.

18. Fletcher KA, Fakayode SO, Lowry M, et al. Molecular fluorescence, phosphorescence, and chemiluminescence spectrometry. Anal Chem. 2006;78(12):4047-4068.

19. Mok H, Jeong H, Kim SJ, Chung BH. Indocyanine green encapsulated nanogels for hyaluronidase activatable and selective near infrared imaging of tumors and lymph nodes. Chem Commun (Camb). 2012;48(69):8628-8630.
20. Kjellman P, Zandt RI, Fredriksson S, Strand SE. Optimizing retention of multimodal imaging nanostructures in sentinel lymph nodes by nanoscale size tailoring. Nanomedicine. 2014;S1549-9634(14):00031-00038.

21. Rogers JM, Jung CW, Lewis J, Groman EV. Use of USPIO-induced magnetic susceptibility artifacts to identify sentinel lymph nodes and lymphatic drainage patterns. I. Dependence of artifact size with subcutaneous Combidex dose in rats. Magn Reson Imaging. 1998;16(8):917-923.

22. Fifis T, Gamvrellis A, Crimeen-Irwin B, et al. Size-dependent immunogenicity: therapeutic and protective properties of nano-vaccines against tumors. J Immunol. 2004;173(5):3148-3154.

23. Oh KS, Song JY, Cho SH, et al. Paclitaxel-loaded Pluronic nanoparticles formed by a temperature-induced phase transition for cancer therapy. $J$ Control Release. 2010;148(3):344-350.

24. Yuk SH, Oh KS, Park J, Lee EH, Kim K, Kwon IC. Docetaxel-loaded composite nanoparticles formed by a temperature-induced phase transition for cancer therapy. $J$ Bioact Compat Polym. 2012;27(5): 441-452.

25. Kraynak MA. Pharmaceutical aspects of docetaxel. Am J Health Syst Pharm. 1997;54(24 Suppl 2):S7-S10.

26. Oussoren C, Zuidema J, Crommelin DJ, Storm G. Lymphatic uptake and biodistribution of liposomes after subcutaneous injection. II. Influence of liposomal size, lipid compostion and lipid dose. Biochim Biophys Acta. 1997;1328(2):261-272.

27. Hawley AE, Illum L, Davis SS. Lymph node localisation of biodegradable nanospheres surface modified with poloxamer and poloxamine block co-polymers. FEBS Lett. 1997;400(3):319-323.

28. Ali Khan A, Mudassir J, Mohtar N, Darwis Y. Advanced drug delivery to the lymphatic system: lipid-based nanoformulations. Int J Nanomedicine. 2013;8(1):2733-2744.

29. Ryu JH, Na JH, Ko HK, et al. Non-invasive optical imaging of cathepsin B with activatable fluorogenic nanoprobes in various metastatic models. Biomaterials. 2014;35(7):2302-2311.

30. Choi KY, Jeon EJ, Yoon HY, et al. Theranostic nanoparticles based on PEGylated hyaluronic acid for the diagnosis, therapy and monitoring of colon cancer. Biomaterials. 2012;33(26):6186-6193.

31. Yhee JY, Lee SJ, Lee S, et al. Tumor-targeting transferrin nanoparticles for systemic polymerized siRNA delivery in tumor-bearing mice. Bioconjug Chem. 2013;24(11):1850-1860.

32. Na JH, Koo H, Lee S, et al. Real-time and non-invasive optical imaging of tumor-targeting glycol chitosan nanoparticles in various tumor models. Biomaterials. 2011;32(22):5252-5261.

33. Liu J, Yu M, Zhou C, Yang S, Ning X, Zheng J. Passive tumor targeting of renal-clearable luminescent gold nanoparticles: long tumor retention and fast normal tissue clearance. J Am Chem Soc. 2013; 135(13):4978-4981.

34. Kumar R, Roy I, Ohulchanskky TY, et al. In vivo biodistribution and clearance studies using multimodal organically modified silica nanoparticles. ACS Nano. 2010;4(2):699-708.

35. Wang B, He X, Zhang Z, Zhao Y, Feng W. Metabolism of nanomaterials in vivo: blood circulation and organ clearance. Acc Chem Res. 2013;46(3):761-769.

36. Cho YW, Lee J, Lee SC, Huh KM, Park K. Hydrotropic agents for study of in vitro paclitaxel release from polymeric micelles. J Control Release. 2004;97(2):249-257.
International Journal of Nanomedicine

\section{Publish your work in this journal}

The International Journal of Nanomedicine is an international, peerreviewed journal focusing on the application of nanotechnology in diagnostics, therapeutics, and drug delivery systems throughout the biomedical field. This journal is indexed on PubMed Central, MedLine, CAS, SciSearch ${ }^{\circledR}$, Current Contents ${ }^{\circledR} /$ Clinical Medicine,

\section{Dovepress}

Journal Citation Reports/Science Edition, EMBase, Scopus and the Elsevier Bibliographic databases. The manuscript management system is completely online and includes a very quick and fair peer-review system, which is all easy to use. Visit http://www.dovepress.com/ testimonials.php to read real quotes from published authors. 